\title{
Recent studies of CP violation in bottom meson decays at Belle
}

\author{
P. Vanhoefer** \\ Max-Planck-Institut für Physik, 80805 München \\ E-mail: pvanhoef@mpp.mpg.de
}

We present a summary of recent studies on $C P$ violation with the Belle experiment using the final data sample of $772 \times 10^{6} B \bar{B}$ pairs produced at the $\Upsilon(4 S)$ resonance at the KEK asymmetric $e^{+} e^{-}$ collider. We discuss preliminary measurements of the branching fraction, the polarization and the $C P$ asymmetries of the decay $B^{0} \rightarrow \rho^{+} \rho^{-}$and an updated constraint on the CKM angle $\phi_{2}$ from the $B \rightarrow \rho \rho$ system. Being also related to $\phi_{2}$, we present a preliminary measurement of the branching fraction of $B^{0} \rightarrow \pi^{0} \pi^{0}$ decays. Last, a preliminary model independent dalitz plot analysis of the decay $B^{0} \rightarrow D^{0}\left[K_{S}^{0} \pi^{+} \pi^{-}\right] K^{* 0}$ is presented and its sensitivity to the CKM angle $\phi_{3}$ is discussed.

The European Physical Society Conference on High Energy Physics 22-29 July 2015

Vienna, Austria

* Speaker.

$\dagger$ for the Belle Collaboration 


\section{Introduction}

Testing the predictions of the Cabibbo-Kobayashi-Maskawa (CKM) mechanism for violation of the combined charge-parity $(C P)$ symmetry $[1,2]$ is one of the major precision tests [3-5] of the flavor sector of the Standard-Model (SM). The Belle experiment at KEK significantly contributed to the validation of the CKM scheme and to constraining the unitarity triangle for $B$ decays to its current precision. Up-to-date, the SM describes the data impressively well, but there is still room for a deviation from unitarity, which clearly would point towards physics beyond the SM. These proceedings give a summary of the experimental status of measurements related to two of the CKM angles defined from the CKM matrix elements as: $\phi_{2} \equiv \arg \left(V_{t d} V_{t b}^{*}\right) /\left(-V_{u d} V_{u b}^{*}\right)$ and $\phi_{3} \equiv \arg \left(V_{u d} V_{u b}^{*}\right) /\left(-V_{c d} V_{c b}^{*}\right)$. All measurements presented here are based on Belle's final data set of $772 \times 10^{6} B \bar{B}$ pairs.

\section{The CKM Angle $\phi_{2}$}

The CKM angle $\phi_{2}$ can be determined by measuring the time-dependent asymmetry between $B^{0}$ and $\bar{B}^{0}$ decays into a common $C P$ eigenstate [6] made out of unflavored quarks ( $\bar{b} \rightarrow \bar{u} u \bar{d}$ quark transitions). Examples are the decays $B^{0} \rightarrow \pi \pi, \rho \pi, \rho \rho$ and $a_{1}(1260) \pi$ [7-14]. In the decay sequence, $\Upsilon(4 S) \rightarrow B_{C P} B_{\mathrm{Tag}} \rightarrow f_{C P} f_{\mathrm{Tag}}$, where one of the $B$ mesons decays into a $C P$ eigenstate $f_{C P}$ at a time $t_{C P}$ and the other decays into a flavor specific final state $f_{\text {Tag }}$ at a time $t_{\text {Tag }}$, the timedependent decay rate is given by

$$
P(\Delta t, q)=\frac{e^{-|\Delta t| / \tau_{B^{0}}}}{4 \tau_{B^{0}}}\left[1+q\left(A_{C P} \cos \Delta m_{d} \Delta t+S_{C P} \sin \Delta m_{d} \Delta t\right)\right],
$$

where $\Delta t \equiv t_{C P}-t_{\text {Tag }}$ is the lifetime difference between the two $B$ mesons, $\Delta m_{d}$ is the mass difference between the mass eigenstates $B_{H}$ and $B_{L}$ and $q=+1(-1)$ for $B_{\mathrm{Tag}}=B^{0}\left(\bar{B}^{0}\right)$. The $C P$ asymmetry is given by

$$
\frac{N\left(\bar{B} \rightarrow f_{C P}\right)-N\left(B \rightarrow f_{C P}\right)}{N\left(\bar{B} \rightarrow f_{C P}\right)+N\left(B \rightarrow f_{C P}\right)}
$$

where $N\left(B(\bar{B}) \rightarrow f_{C P}\right)$ is the number of events of a $B(\bar{B})$ decaying to $f_{C P}$, the asymmetry can be time-dependent. The parameters $A_{C P}$ and $S_{C P}$ describe direct and mixing-induced $C P$ violation, respectively ${ }^{1}$.

At tree level one expects $A_{C P}=0$ and $S_{C P}=\sin 2 \phi_{2}$ for the above mentioned decays sensitive to $\phi_{2}$. Possible penguin contributions can give rise of direct $C P$ violation, $A_{C P} \neq 0$ and also pollute the measurement of $\phi_{2}, S_{C P}=\sqrt{1-A_{C P}^{2}} \sin \left(2 \phi_{2}^{\text {eff }}\right)$ where the observed $\phi_{2}^{\text {eff }} \equiv \phi_{2}-\Delta \phi_{2}$ is shifted by $\Delta \phi_{2}$ due to different weak and strong phases from additional non-leading contributions. Despite this, it is possible to determine $\Delta \phi_{2}$ in $B^{0} \rightarrow h^{+} h^{-}$with an $S U(2)$ isospin analysis by considering the set of three $B \rightarrow h h$ decays where the $h h$ s are either two pions or two longitudinally polarized $\rho s$, related via isospin symmetry [15]. The $B \rightarrow h^{i} h^{j}$ amplitudes $A_{i j}$ obey the triangle relations,

$$
A_{+0}=\frac{1}{\sqrt{2}} A_{+-}+A_{00}, \quad \bar{A}_{-0}=\frac{1}{\sqrt{2}} \bar{A}_{+-}+\bar{A}_{00} .
$$

\footnotetext{
${ }^{1}$ There exists an alternate notation where $C_{C P}=-A_{C P}$.
} 


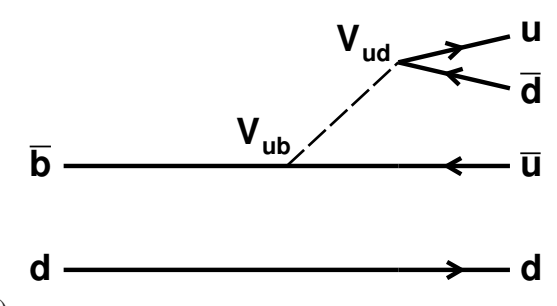

a)

Figure 1: a) leading order and b) penguin feynman diagrams for color-allowed $b \rightarrow u \bar{u} d$ transitions.

Isospin arguments demonstrate that $B^{+} \rightarrow h^{+} h^{0}$ is a pure first-order mode in the limit of neglecting electroweak penguins, thus these triangles share the same base, $A_{+0}=\bar{A}_{-0}$, see Fig. 2 for an illustration. $\Delta \phi_{2}$ can then be determined from the difference between the two triangles. This method has an inherent four-fold discrete ambiguity in the determination of $\sin \left(2 \phi_{2}\right)$.

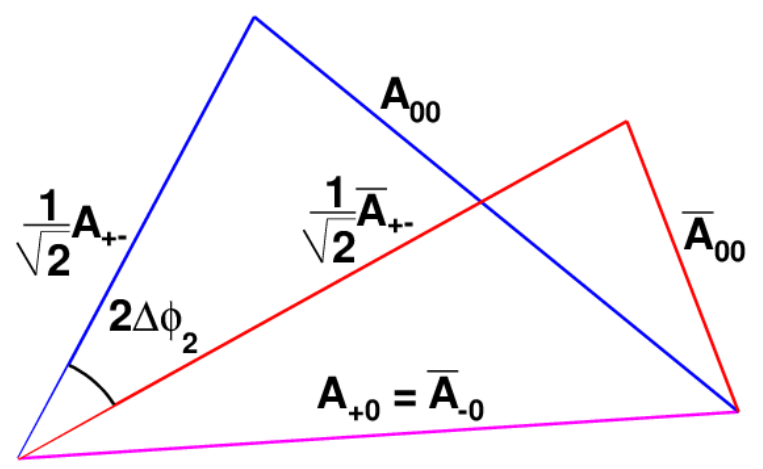

Figure 2: A sketch of the isospin triangle.

\subsection{The Decay $B^{0} \rightarrow \rho^{+} \rho^{-}$}

Having a decay into two vector particles, an angular analysis is performed to separate the $C P$-even states from the $C P$-odd states for the isopsin analysis. Longitudinal polarized states correspond to pure $C P$-even states and their fraction, $f_{L}$, is obtained from a fit to the cosine of helicity angles, $\Theta_{\mathrm{H}}^{ \pm}$, which are defined as sketched in Fig. 3. Previous measurements show that $f_{L}$ is consistent with one [10-12], consequently the isospin analysis is performed for longitudinal polarization only. In addition to combinatorial background, the presence of multiple, largely unknown backgrounds with the same four-pion final state as $B^{0} \rightarrow \rho^{+} \rho^{-}$make this decay quite difficult to isolate and interference between the various $4 \pi$ modes needs to be considered. Besides updating to the full data set, the branching fraction, the fraction of longitudinally polarized $\rho$ mesons on this decay and the $C P$ violating parameters are obtained simultaneously from the fit to the data. In the fit, Belle uses the missing energy $\left(\Delta E \equiv E_{B}^{*}-E_{\text {beam }}^{*}\right)$, the beam-constraint $B$ mass $\left(M_{\mathrm{bc}}=\sqrt{E_{\mathrm{beam}}^{* 2}-p_{B}^{* 2}}\right)$, the masses and helicity angles of the two reconstructed $\rho^{ \pm}$mesons, a fisher discriminant to separate the jet-like $e^{+} e^{-} \rightarrow q \bar{q},(q=u, d, s, c)$ background from the spherical $B \bar{B}$ decays and the $\Delta t$ distribution for the two flavors of $B_{\text {Tag. }}$. They obtain 


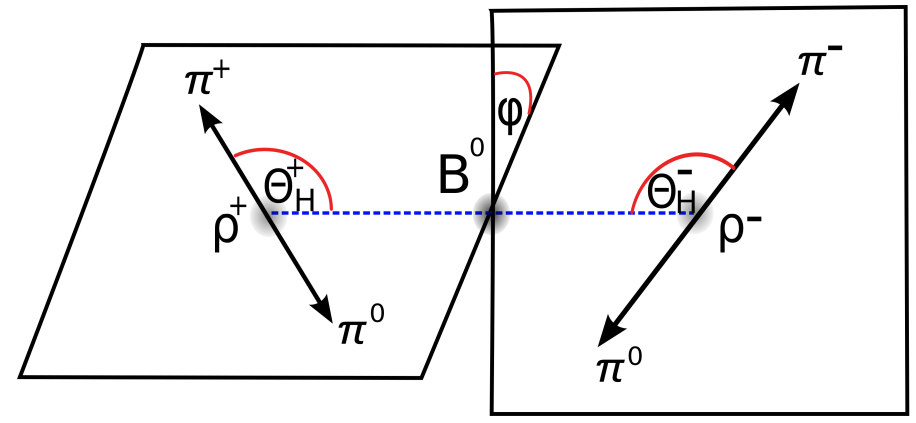

Figure 3: The helicity angles $\cos \Theta_{\mathrm{H}}^{ \pm}$; each one is defined in its $\rho$ rest frame.

$$
\begin{aligned}
& \mathscr{B}\left(B^{0} \rightarrow \rho^{+} \rho^{-}\right)=(28.3 \pm 1.5(\text { stat }) \pm 1.4(\text { syst })) \times 10^{-6} \\
& f_{L}=0.988 \pm 0.012(\text { stat }) \pm 0.023(\text { syst }) \\
& S_{C P}=-0.13 \pm 0.15(\text { stat }) \pm 0.05(\text { syst }) \text { and } \\
& A_{C P}=0.00 \pm 0.10(\text { stat }) \pm 0.06 \text { (syst) }
\end{aligned}
$$

This is currently the most precise measurement of the branching fraction and polarization of $B \rightarrow$ $\rho^{+} \rho^{-}$decays as well as the tightest constraint on $C P$ violation in this mode. Fig. 4 shows the projections onto $\Delta E, \cos \Theta_{\mathrm{H}}^{+}$and onto $\Delta t$ for the two flavor of $B_{\text {Tag }}$, each with the fit result on top. The results from this measurement are used in an isospin analysis together with other Belle
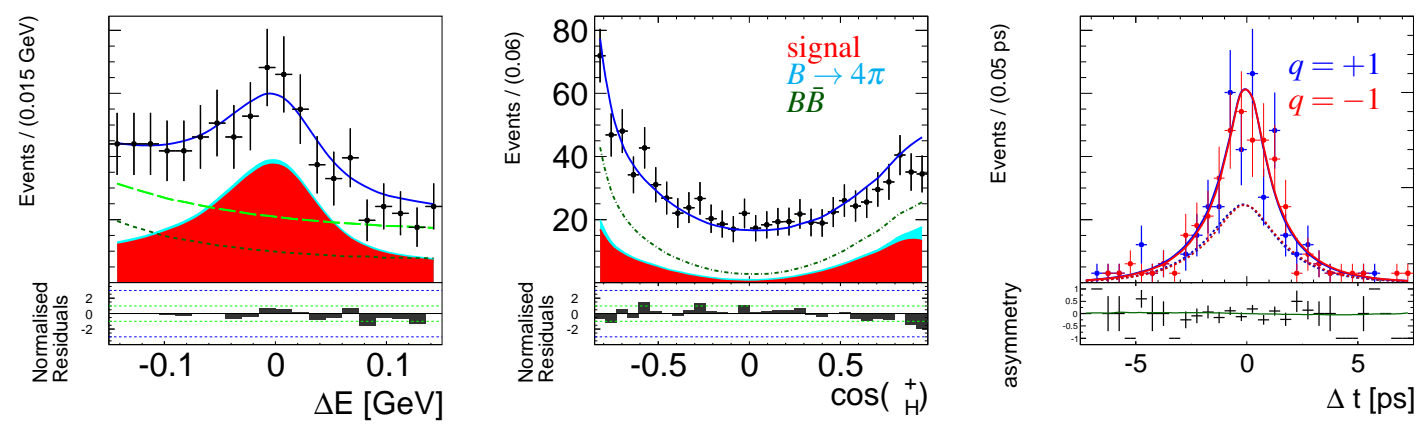

Figure 4: Signal enhanced distributions of (a) $\Delta E$, (b) $\cos \Theta_{H}$, and (c) $\Delta t$ for the two flavors of $B_{\text {Tag }}\left(B_{\text {Tag }}=\right.$ $B^{0}$ for $q=+1$ ) with the fit result on top. The shaded red area is the $B^{0} \rightarrow \rho^{0} \rho^{0}$ contribution. Furthermore, all $B$ decays with a four pion final state are shown in cyan, the entire $(B \bar{B})$ background in dashed (dash-dotted dark) green and the full PDF in blue.

measurements [13,16] (longitudinal polarization only). Fig. 5 b) shows the $\phi_{2}$ scan from the isospin analysis, the constraint most consistent with other measurements of the CKM triangle is $\phi_{2}=$ $(93.7 \pm 10.6)^{\circ}$ and the penguin pollution is consistent with zero: $\Delta \phi_{2}=(0.0 \pm 9.6)^{\circ}$. In the $B \rightarrow \rho \rho$ system, the relatively small amplitude of $B^{0} \rightarrow \rho^{0} \rho^{0}$ makes the isospin triangles flat and therefore the isospin analysis has no ambiguity. 


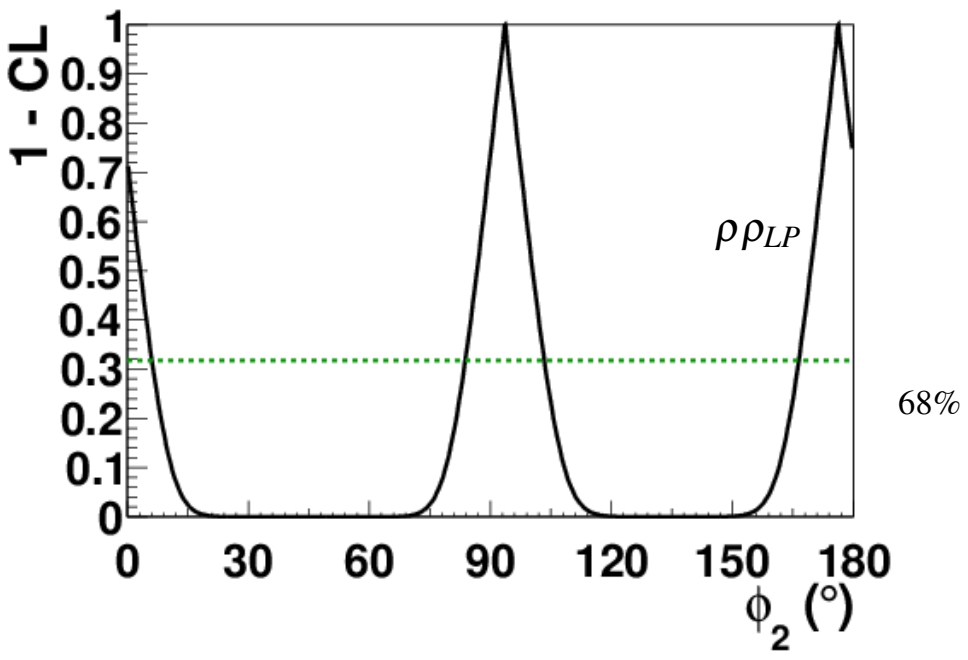

Figure 5: Probability scan of $\phi_{2}$ in the $B \rightarrow \rho \rho$ system.

\subsection{The Decay $B^{0} \rightarrow \pi^{0} \pi^{0}$}

This decay is an important input for the isospin analysis in the $B \rightarrow \pi \pi$ system. Being reconstructed from four $\gamma_{\mathrm{S}}$ makes this measurement experimentally quite challenging. A fit to $\Delta E, M_{\mathrm{bc}}$ and a fisher discriminant $T_{C}$ is performed and a preliminary branching fraction of

$$
\mathscr{B}\left(B \rightarrow \pi^{0} \pi^{0}\right)=(0.9 \pm 0.12 \text { stat } \pm 0.10 \text { syst }) \times 10^{-6}
$$

is obtained. Signal enhanced projections are shown in Fig. 6. It is planned to supersede this currently most precise measurement of the branching fraction with one including the determination of the direct $C P$ violation in this mode.

The upcoming Belle 2 experiment will make a time-dependent analysis possible, as the accumulated data will provides enough data to use converted photons to determine the $B$ vertex. Including the mixing-induced $C P$-violation parameter of $B \rightarrow \pi^{0} \pi^{0}$ in the isospin analysis might remove the four-fold ambiguity from the isospin analysis being currently present in the $B \rightarrow \pi \pi$ system.

\section{The Decay $B^{0} \rightarrow D^{0}\left[K_{S}^{0} \pi^{+} \pi^{-}\right] K^{* 0}$ and the CKM Angle $\phi_{3}$}

A model independent dalitz plot analysis $[17,18]$ of the decay $B^{0} \rightarrow D^{0}\left[K_{S}^{0} \pi^{+} \pi^{-}\right] K^{* 0}$ has been performed for the first time. The flavor-specific decay, $K^{* 0} \rightarrow K^{+} \pi^{-}$, allows to determine the flavor of the $B$ meson. The number of events in different bins of the dalitz plot of the $D$ meson coming from either a $B^{0}\left(N_{i}^{+}\right)$or a $\bar{B}^{0}\left(N_{i}^{-}\right)$is given by

$$
\left.N_{i}^{ \pm}=h_{B}\left[K_{ \pm i}+r_{S}^{2} K_{\mp i}+2 k \sqrt{K_{i} K_{-i}}\left(x_{ \pm} c_{i} \pm y_{ \pm} s_{i}\right)\right)\right],
$$

where $h_{B}$ is a normalization constant, $K_{ \pm i}$ are the entries in the $D(+)$ or $\bar{D}(-)$ dalitz plot, $k=$ $0.95 \pm 0.03$ [20] accounts for interference effects in the $D$ decay, and $c_{i}$ and $s_{i}$ include information on the average of the phase variation within a dalitz plot bin. All information on the $D$ dalitz plot is 

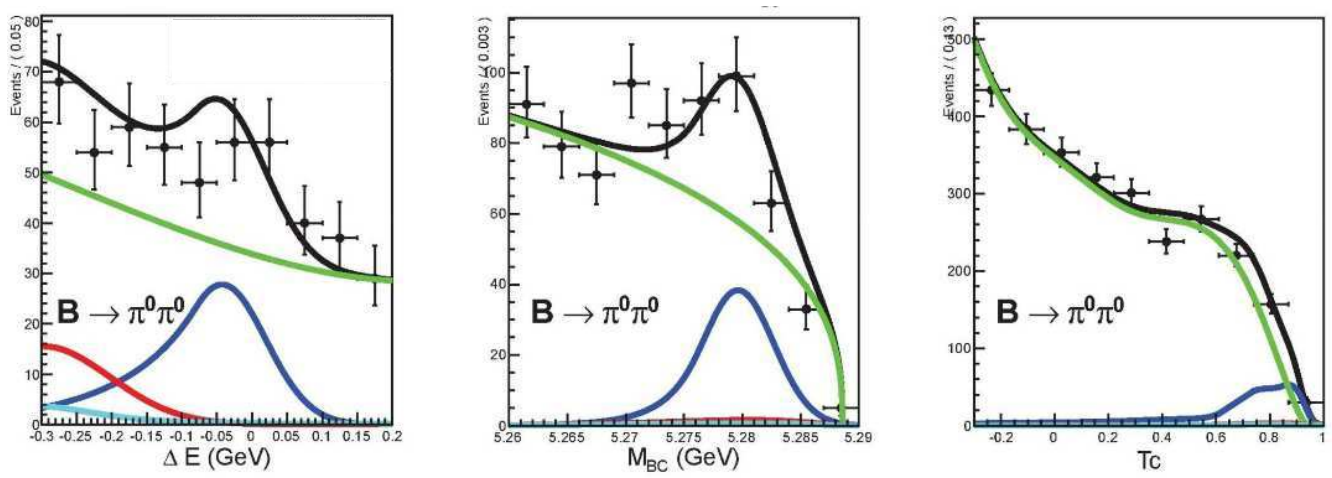

Figure 6: Signal enhanced distributions of (a) $\Delta E$, (b) $\cos \Theta_{H}$ and (c) $\Delta t$ with the fit result on top. Contributions from signal, continuum, $\rho \pi$ and other rare B decays are shown by blue, green, red and cyan respectively.

provided by measurements from the CLEO collaboration [19]. The observables $x_{ \pm} \equiv r_{S} \cos \left(\delta_{S} \pm\right.$ $\left.\phi_{3}\right)$ and $y_{ \pm} \equiv r_{s} \sin \left(\delta_{S} \pm \phi_{3}\right)$ from the interference term in Equ. 3.1 allow an extraction of the CKM angle $\phi_{3}$ in general, where the ratio between the cabbibo-allowed and double-cabbibo-suppressed amplitudes, $r_{S} \equiv \frac{\bar{A}}{A}=\frac{A\left(\bar{B} \rightarrow \bar{D}^{0} \bar{K}^{* 0}\right)}{A\left(\bar{B} \rightarrow D^{0} \bar{K}^{* 0}\right)}$, indicates the sensitivity to $\phi_{3}$. Belle obtains

$$
\begin{array}{ll}
x_{+}=+0.1_{-0.4-0.1}^{+0.7+0.0} \pm 0.1, & y_{+}=+0.3_{-0.8-0.1}^{+0.5+0.0} \pm 0.1, \\
x_{-}=+0.4_{-0.8-0.1}^{+0.5+0.0} \pm 0.0, & y_{-}=-0.6_{-1.0-0.0}^{+0.8+0.1} \pm 0.1,
\end{array}
$$

and uses the result to obtain an upper limit of

$$
r_{S}<0.87
$$

at the one $\sigma$ level. The (dalitz plot integrated) fit results are shown in Fig. 7 and the $r_{S}$ scan is shown in Fig. 8. This mode is still statistically limited but will give additional insights on $\phi_{3}$ when the Belle 2 data will be available.
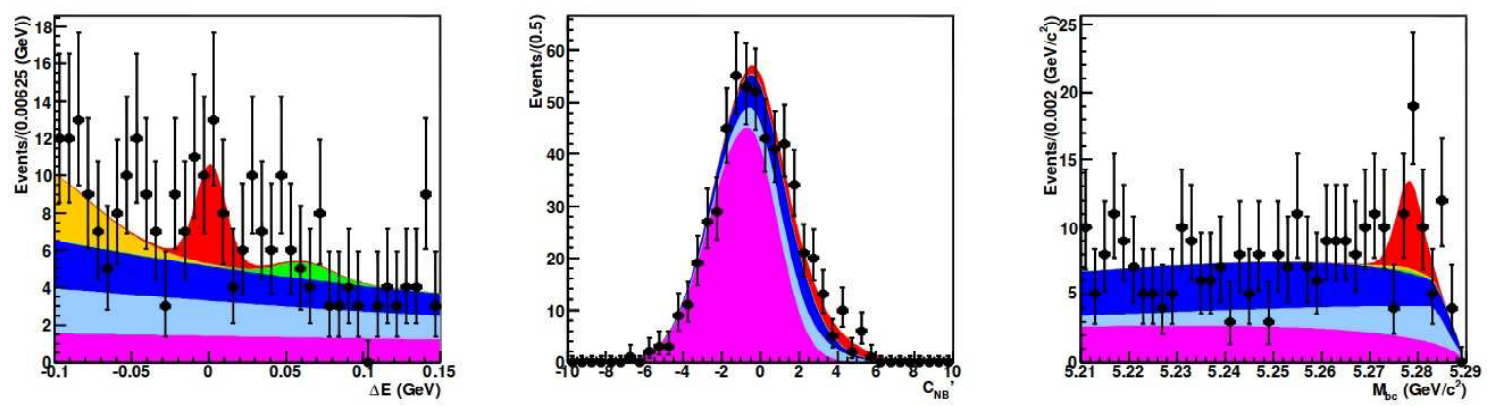

Figure 7: Signal enhanced distributions of (a) $\Delta E$, (b) $\cos \Theta_{H}$ and (c) $\Delta t$ with the fit result on top. The signal contribution is shown in red.

\section{Summary}

We have presented three recent and preliminary measurements from Belle sensitive to the CKM phases $\phi_{2}$ and $\phi_{3}$ using the full data set of 772 million $B \bar{B}$ pairs. Measurements of the 


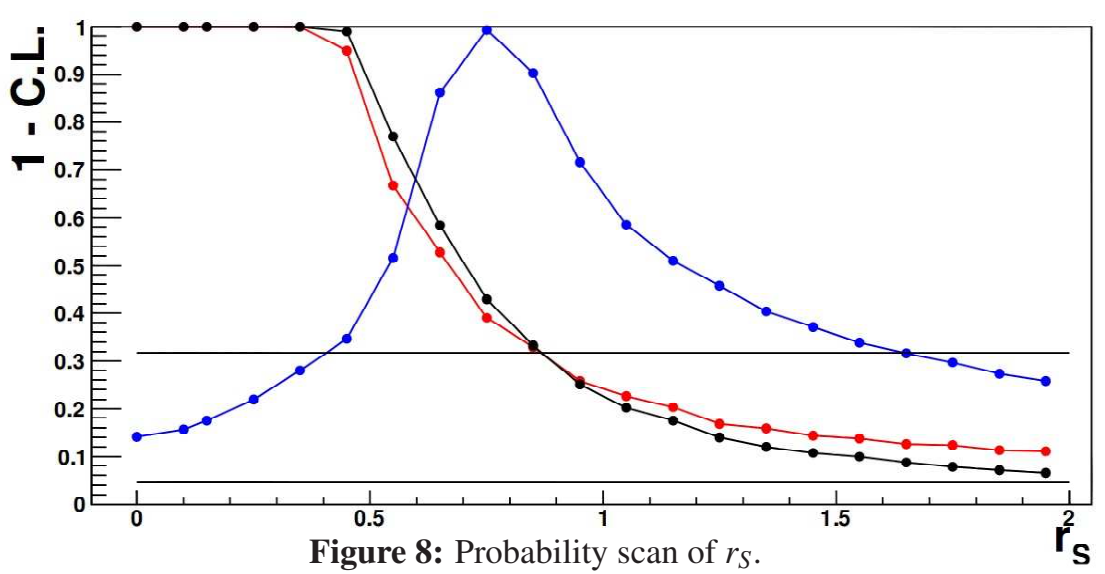

branching fraction, the polarization and the $C P$ asymmetries in $B \rightarrow \rho^{+} \rho^{-}$were used to update the $\phi_{2}$ isospin constraint from Belle. The branching fraction measurement of of $B \rightarrow \pi^{0} \pi^{0}$ has been presented and the importance for this mode the isospin analysis in the $B \rightarrow \pi \pi$ system has been discussed. The current world averages of $\phi_{2}$ as computed by the CKMfitter [21] and UTfit [22] collaborations are $\phi_{2}=\left(87.6_{-3.3}^{+3.5}\right)^{\circ}$ and $\phi_{2}=(88 / 6 \pm 3.3)^{\circ}$, respectively. Furthermore we presented a preliminary measurement of $B^{0} \rightarrow D^{0}\left[K_{S}^{0} \pi^{+} \pi^{-}\right] K^{* 0}$ decays and discussed the sensitivity to $\phi_{3}$. All shown results are in good agreement with other SM based constraints on the CKM triangle. With Belle 2 being built [23] and the LHCb operating, the next generation of $B$ physics experiments are expected to further reduce the uncertainty of the CKM observables and might reveal new phenomena.

\section{References}

[1] N. Cabibbo, Phys. Rev. Lett. 10, 531 (1963).

[2] M. Kobayashi and T. Maskawa, Prog. Theor. Phys. 49, 652 (1973).

[3] H. Boos, J. Reuter and T. Mannel, Phys. Rev. D 70, 036006 (2004).

[4] I. Adachi et al. (The Belle Collaboration), Phys. Rev. Lett. 108, 171802 (2012)

[5] I.I. Bigi and A.I. Sander, , Nucl. Phys. B193, 85 (1981).

[6] I. Bigi and A. Sanda, CP Violation, Cambridge University Press, Cambridge (2009).

[7] J.Dalseno et al. (Belle Collab.), Phys. Rev. D 88, 092003 (2013).

[8] J. P. Lees et al. (BaBar Collab.), Phys. Rev. D 87, 052009 (2013).

[9] ,R. Aaij et al. (LHCb Collab.) JHEP 1310183 (2013).

[10] B. Aubert et al. (BaBar Collab.), Phys. Rev. D 76, 052007 (2007).

[11] A. Somov et al. (Belle Collab.), Phys. Rev. Lett. 96, 171801 (2006).

[12] A. Somov et al. (Belle Collab.), Phys. Rev. D 76, 011104 (2007).

[13] P.Vanhoefer et al. (Belle Collab.), Phys. Rev. D 89, 072008 (2014).

[14] B. Aubert et al. (BaBar Collab.), Phys. Rev. D 78, 071104 (2008). 
[15] M. Gronau and D. London, Phys. Rev. Lett. 65, 3381 (1990).

[16] J. Zhang et al. (Belle Collab.), Phys. Rev. L 91, 221801 (2006).

[17] A. Giri, Y. Grossman, A. Soffer, and J. Zupan, Phys. Rev. D 68, 054018 (2003).

[18] H. Aihara et al., (Belle Collab.), Phys. Rev. D 85, 112014 (2012).

[19] J.Libby, et al. (CLEO Collab.) Phys.Rev. D 82 (2010).

[20] B. Aubert et al. (BaBar Collab.), Phys. Rev. D 79, 072003 (2009).

[21] http://ckmfitter.in2p3.fr

[22] http://www.utfit.org

[23] T. Aushev et al., arXiv:1002.5012 (2010). 